\title{
Supporting Information for Extension of the UNRES Coarse-Grained Force Field to Membrane Proteins in the Lipid Bilayer
}

\author{
Karolina Zięba ${ }^{1}$, Magdalena Ślusarz ${ }^{1}$, Rafał Ślusarz ${ }^{1}$, Adam Liwo ${ }^{1}$, Cezary Czaplewski ${ }^{1}$, \\ Adam K. Sieradzan, ${ }^{1, *}$
}

${ }^{1}$ Faculty of Chemistry, University of Gdańsk, Wita Stwosza 63, 80-308 Gdańsk, Poland

*Corresponding author; phone: +48585235350; fax: +48585235350; e-mail: adasko@sun1.chem.univ.gda.pl 
Table S1. The lipid-phase well depths $\left(4 \epsilon_{L}^{\circ} ; k c a l / m o l\right.$, lower triangle) and the water-phase well depths $\left(4 \epsilon_{w}^{\circ} ; \mathrm{kcal} / \mathrm{mol}\right.$, upper triangle; data from Liwo et al., J. Comput. Chem., 1997, $18,874-887)$ of the side-chain-interaction $\left(U_{S C_{i} S C_{j}}\right)$ potentials.

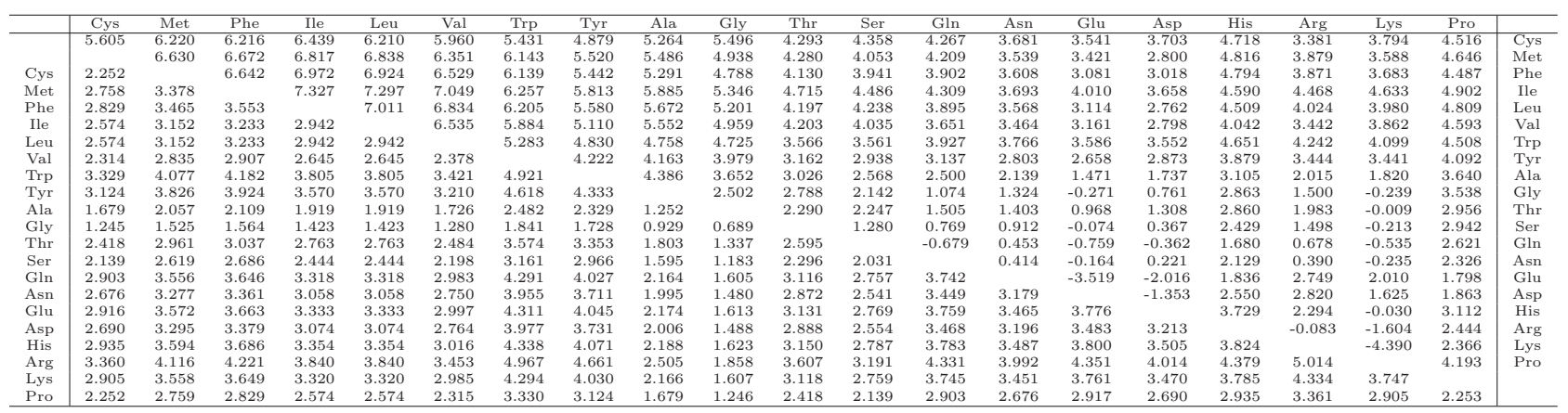




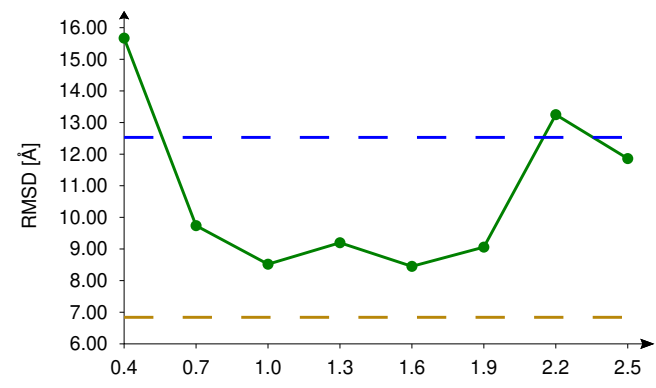

A

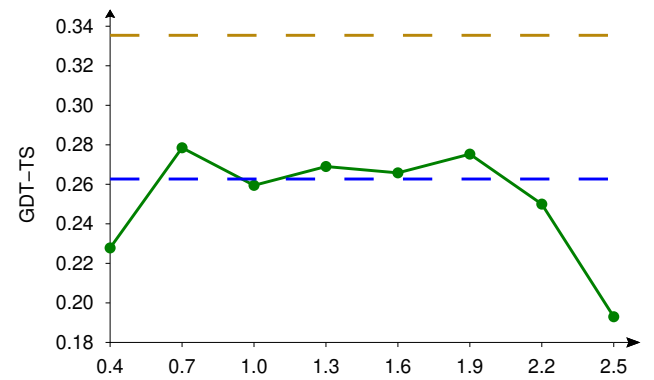

$\mathrm{B}$

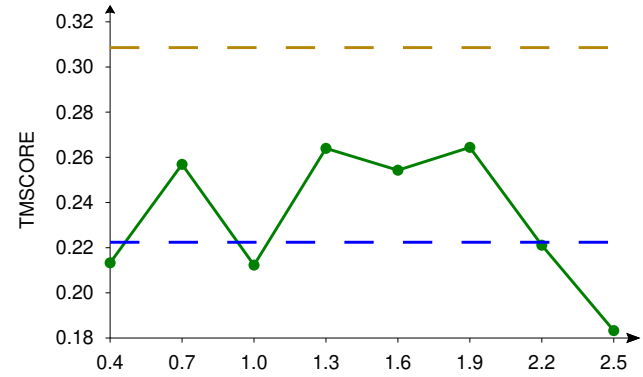

C

Figure S1. Plots of RMSD (A), GDT_TS (B), and TM-score (C) as functions of the $s_{L}$ parameter (eq 9 of the main text) of the lowest-RMSD family of conformations of 1A91 obtained with $\Delta G_{p_{i}}=0 \mathrm{kcal} / \mathrm{mol}$. The values corresponding to all-water and all-lipid environments are as yellow and blue dashed lines respectively. 


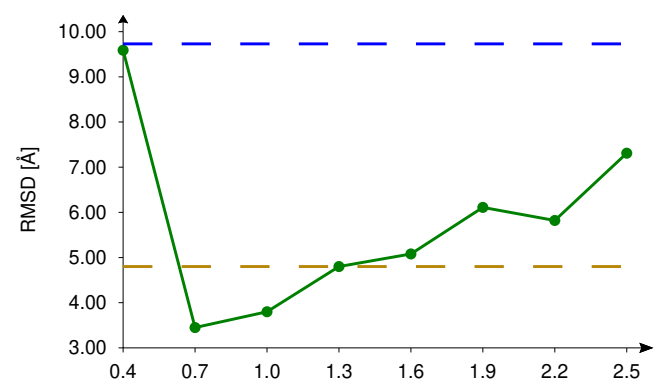

A

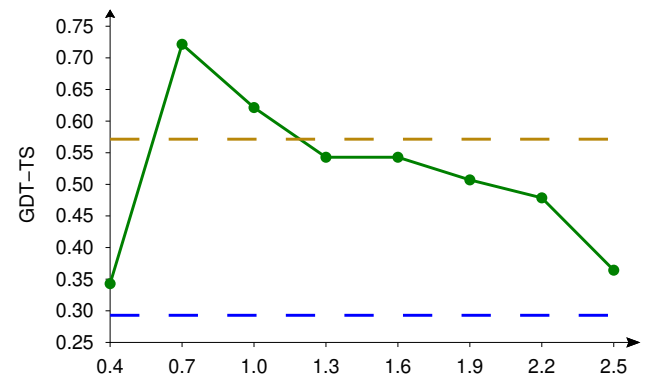

B

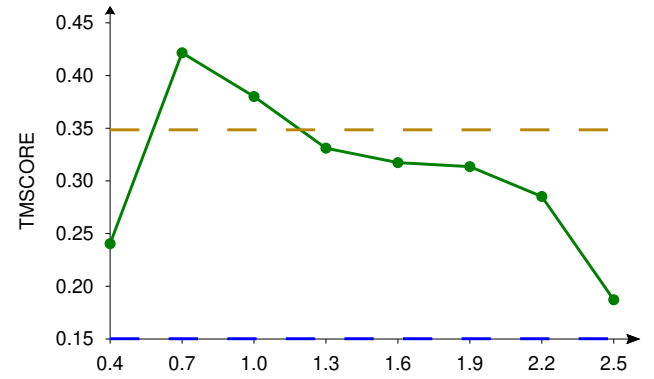

C

Figure S2. Plots of RMSD (A), GDT_TS (B), and TM-score (C) as functions of the $s_{L}$ parameter (eq 9 of the main text) of the lowest-RMSD family of conformations of 1IIJ obtained with $\Delta G_{p_{i}}=0 \mathrm{kcal} / \mathrm{mol}$. The values corresponding to all-water and all-lipid environments are as yellow and blue dashed lines respectively. 


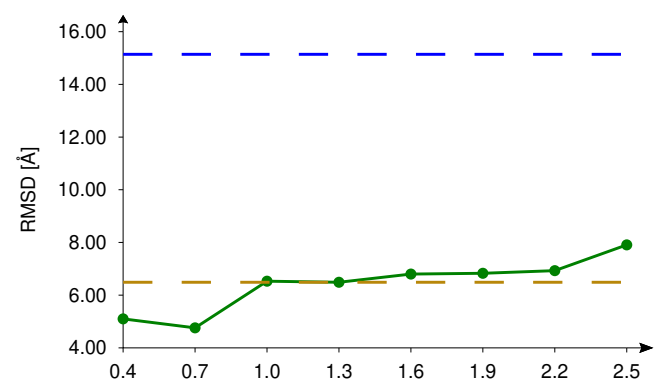

A

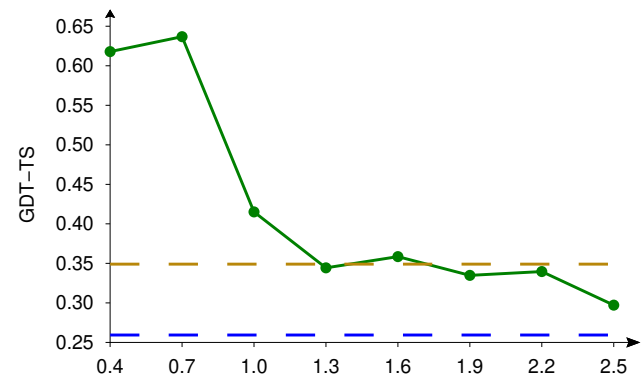

$\mathrm{B}$

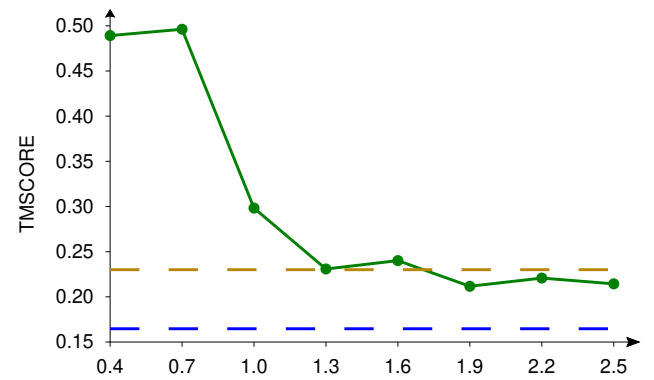

C

Figure S3. Plots of RMSD (A), GDT_TS (B), and TM-score (C) as functions of the $s_{L}$ parameter (eq 9 of the main text) of the lowest-RMSD family of conformations of 1N71 obtained with $\Delta G_{p_{i}}=0 \mathrm{kcal} / \mathrm{mol}$. The values corresponding to all-water and all-lipid environments are as yellow and blue dashed lines respectively. 


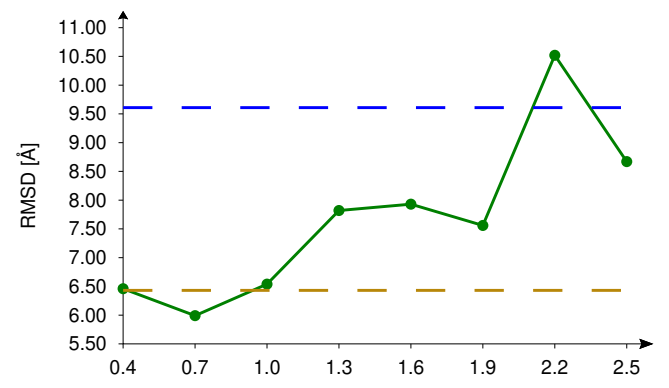

A

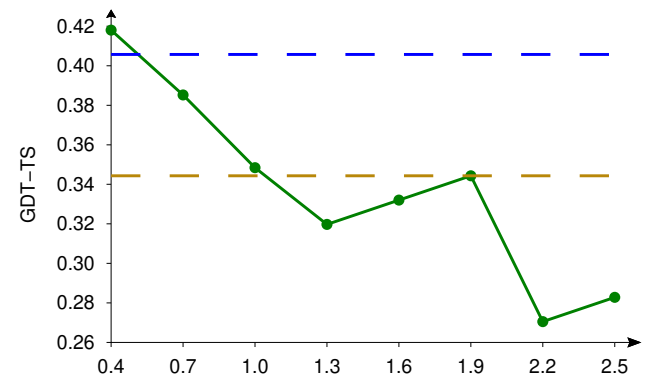

B

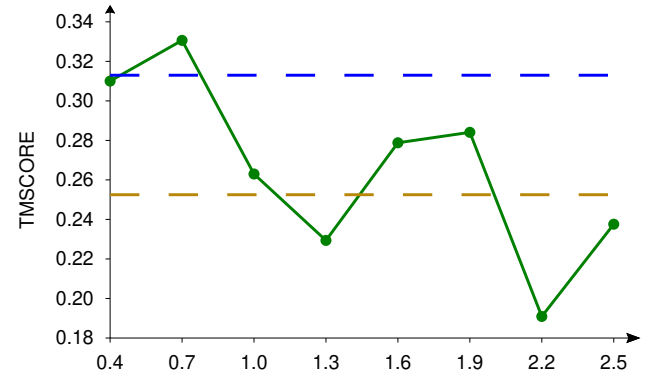

C

Figure S4. Plots of RMSD (A), GDT_TS (B), and TM-score (C) as functions of the $s_{L}$ parameter (eq 9 of the main text) of the lowest-RMSD family of conformations of 1VRY obtained with $\Delta G_{p_{i}}=0 \mathrm{kcal} / \mathrm{mol}$. The values corresponding to all-water and all-lipid environments are as yellow and blue dashed lines respectively. 


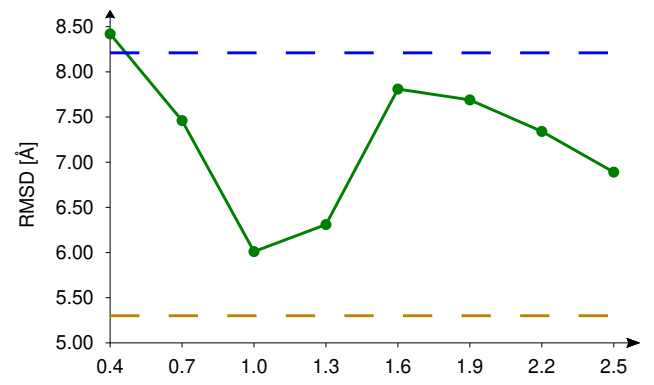

A

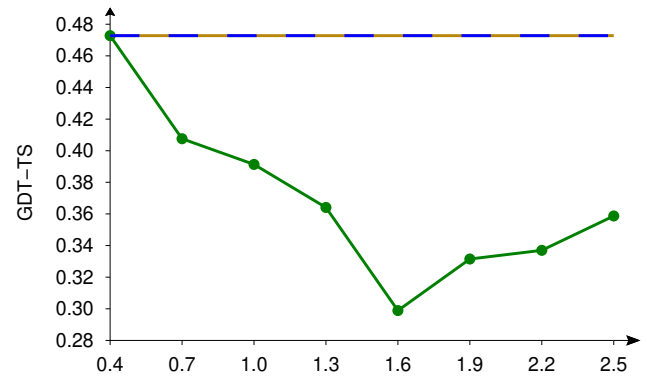

$\mathrm{B}$

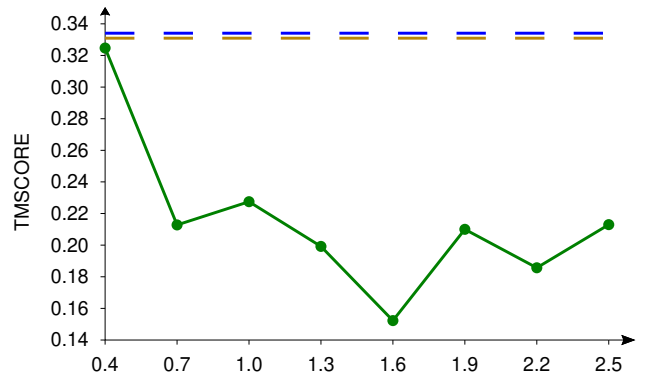

C

Figure S5. Plots of RMSD (A), GDT_TS (B), and TM-score (C) as functions of the $s_{L}$ parameter (eq 9 of the main text) of the lowest-RMSD family of conformations of 1WAZ obtained with $\Delta G_{p_{i}}=0 \mathrm{kcal} / \mathrm{mol}$. The values corresponding to all-water and all-lipid environments are as yellow and blue dashed lines respectively. 


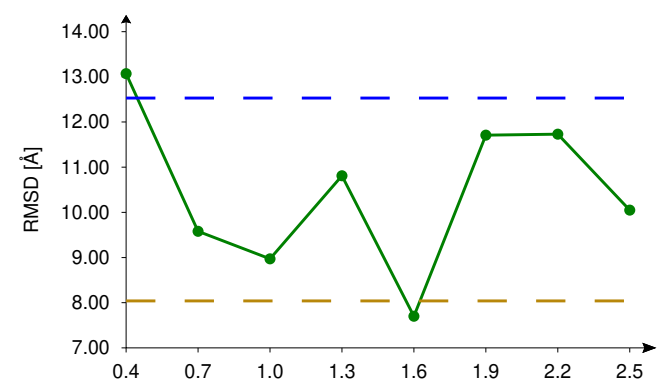

A

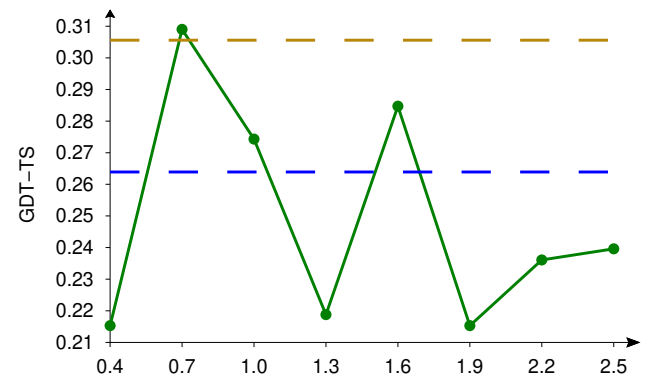

B

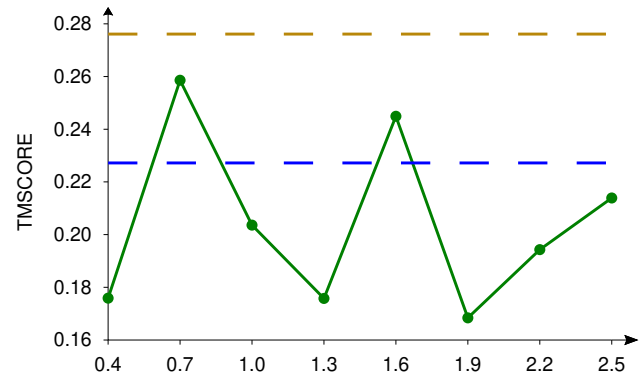

C

Figure S6. Plots of RMSD (A), GDT_TS (B), and TM-score (C) as functions of the $s_{L}$ parameter (eq 9 of the main text) of the lowest-RMSD family of conformations of 1WU0 obtained with $\Delta G_{p_{i}}=0 \mathrm{kcal} / \mathrm{mol}$. The values corresponding to all-water and all-lipid environments are as yellow and blue dashed lines respectively. 


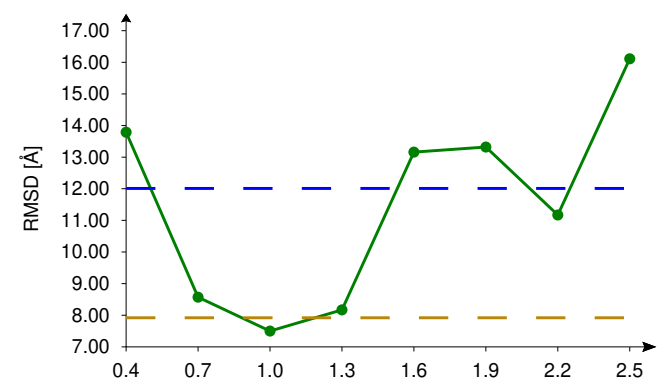

A

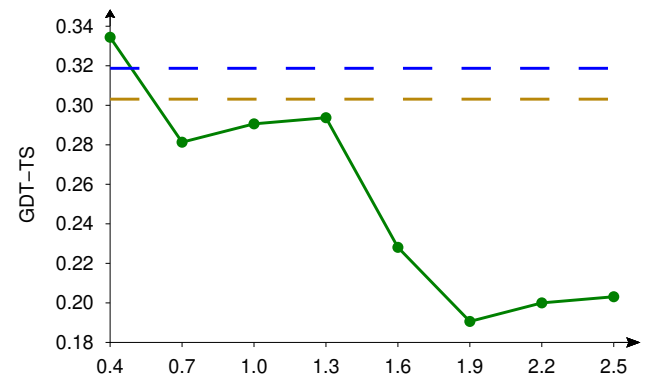

B

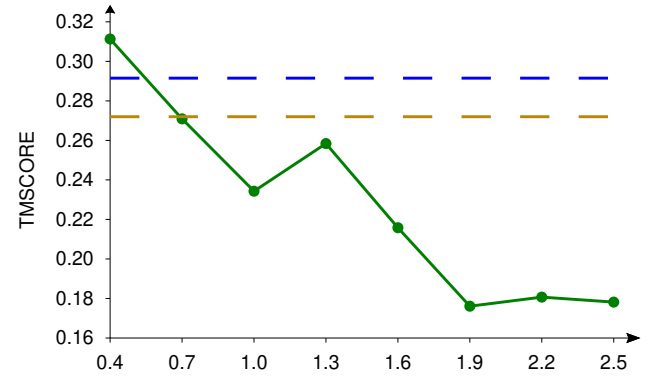

C

Figure S7. Plots of RMSD (A), GDT_TS (B), and TM-score (C) as functions of the $s_{L}$ parameter (eq 9 of the main text) of the lowest-RMSD family of conformations of $2 \mathrm{~K} 9 \mathrm{P}$ obtained with $\Delta G_{p_{i}}=0 \mathrm{kcal} / \mathrm{mol}$. The values corresponding to all-water and all-lipid environments are as yellow and blue dashed lines respectively. 


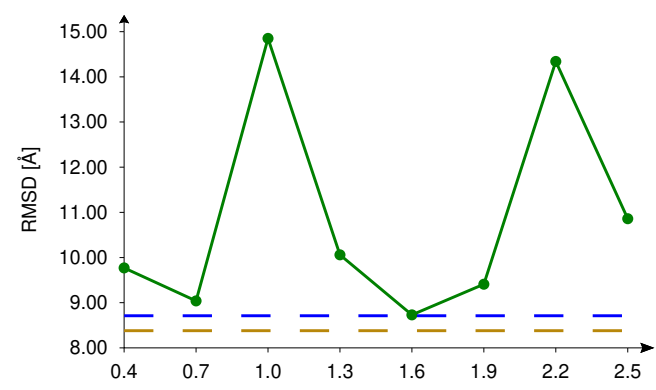

A

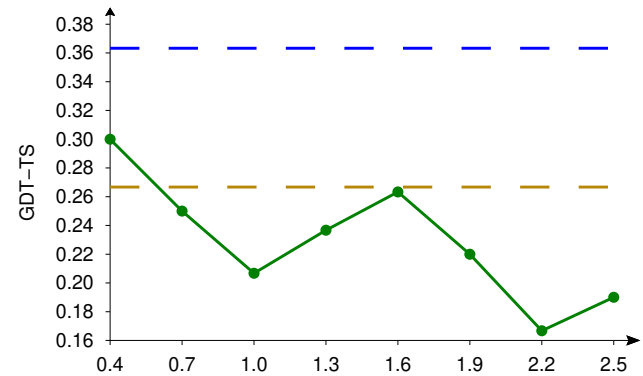

$\mathrm{B}$

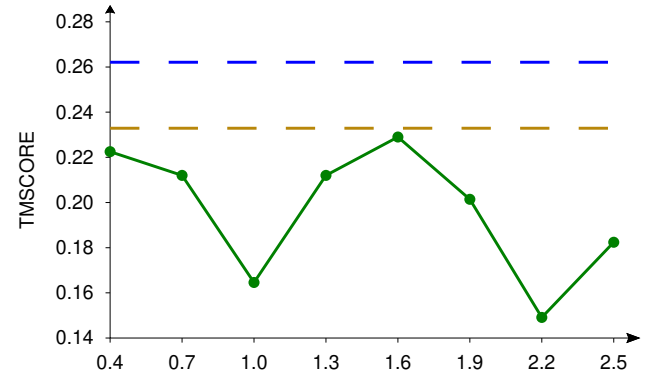

C

Figure S8. Plots of RMSD (A), GDT_TS (B), and TM-score (C) as functions of the $s_{L}$ parameter (eq 9 of the main text) of the lowest-RMSD family of conformations of 2KSD obtained with $\Delta G_{p_{i}}=0 \mathrm{kcal} / \mathrm{mol}$. The values corresponding to all-water and all-lipid environments are as yellow and blue dashed lines respectively. 


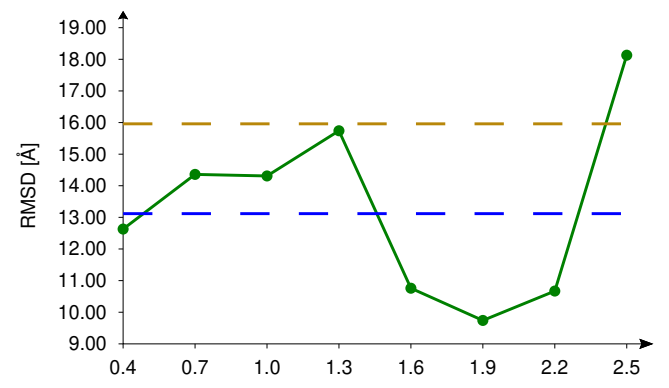

A

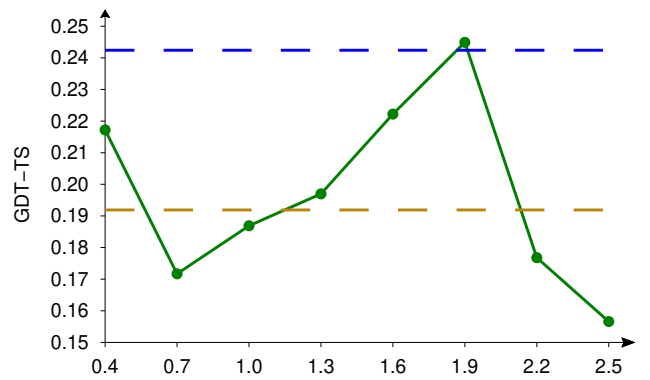

$\mathrm{B}$

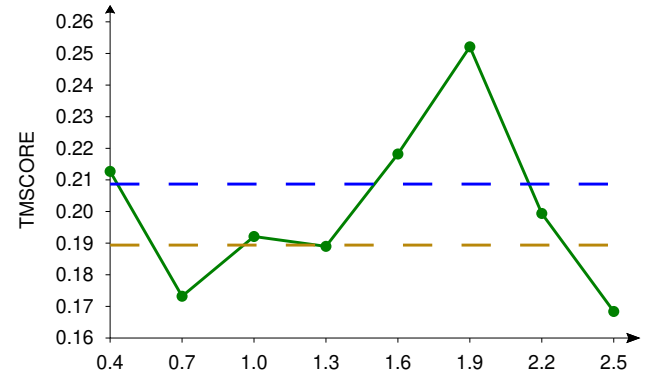

C

Figure S9. Plots of RMSD (A), GDT_TS (B), and TM-score (C) as functions of the $s_{L}$ parameter (eq 9 of the main text) of the lowest-RMSD family of conformations of 2LOP obtained with $\Delta G_{p_{i}}=0 \mathrm{kcal} / \mathrm{mol}$. The values corresponding to all-water and all-lipid environments are as yellow and blue dashed lines respectively. 


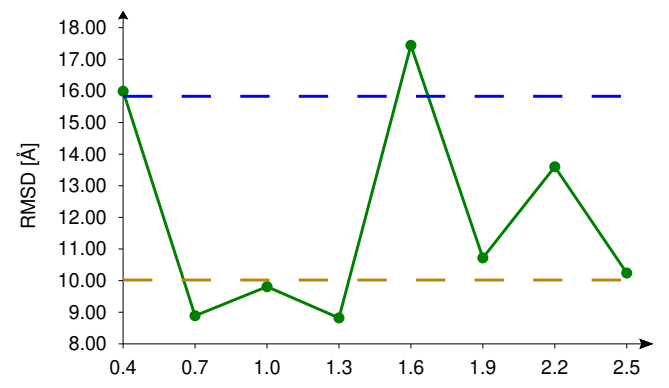

A

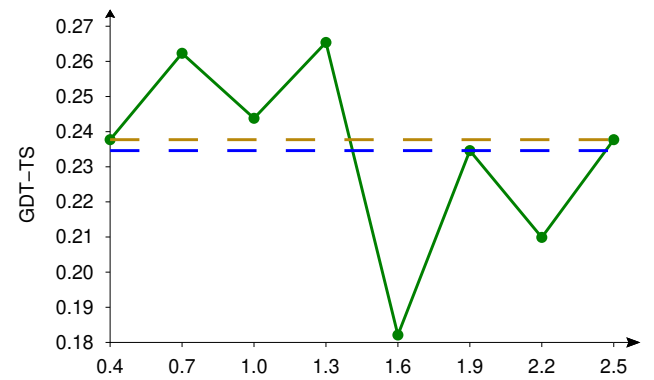

B

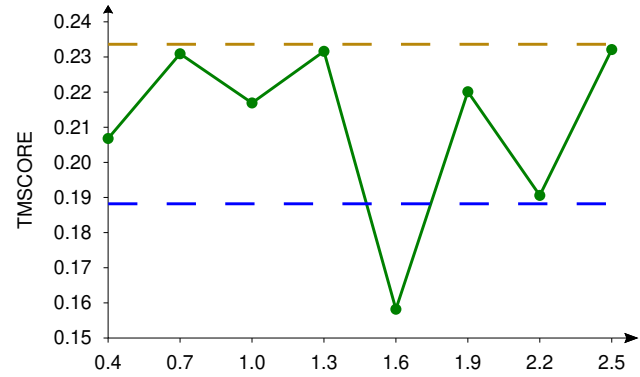

C

Figure S10. Plots of RMSD (A), GDT_TS (B), and TM-score (C) as functions of the $s_{L}$ parameter (eq 9 of the main text) of the lowest-RMSD family of conformations of 2MOZ obtained with $\Delta G_{p_{i}}=0 \mathrm{kcal} / \mathrm{mol}$. The values corresponding to all-water and all-lipid environments are as yellow and blue dashed lines respectively. 


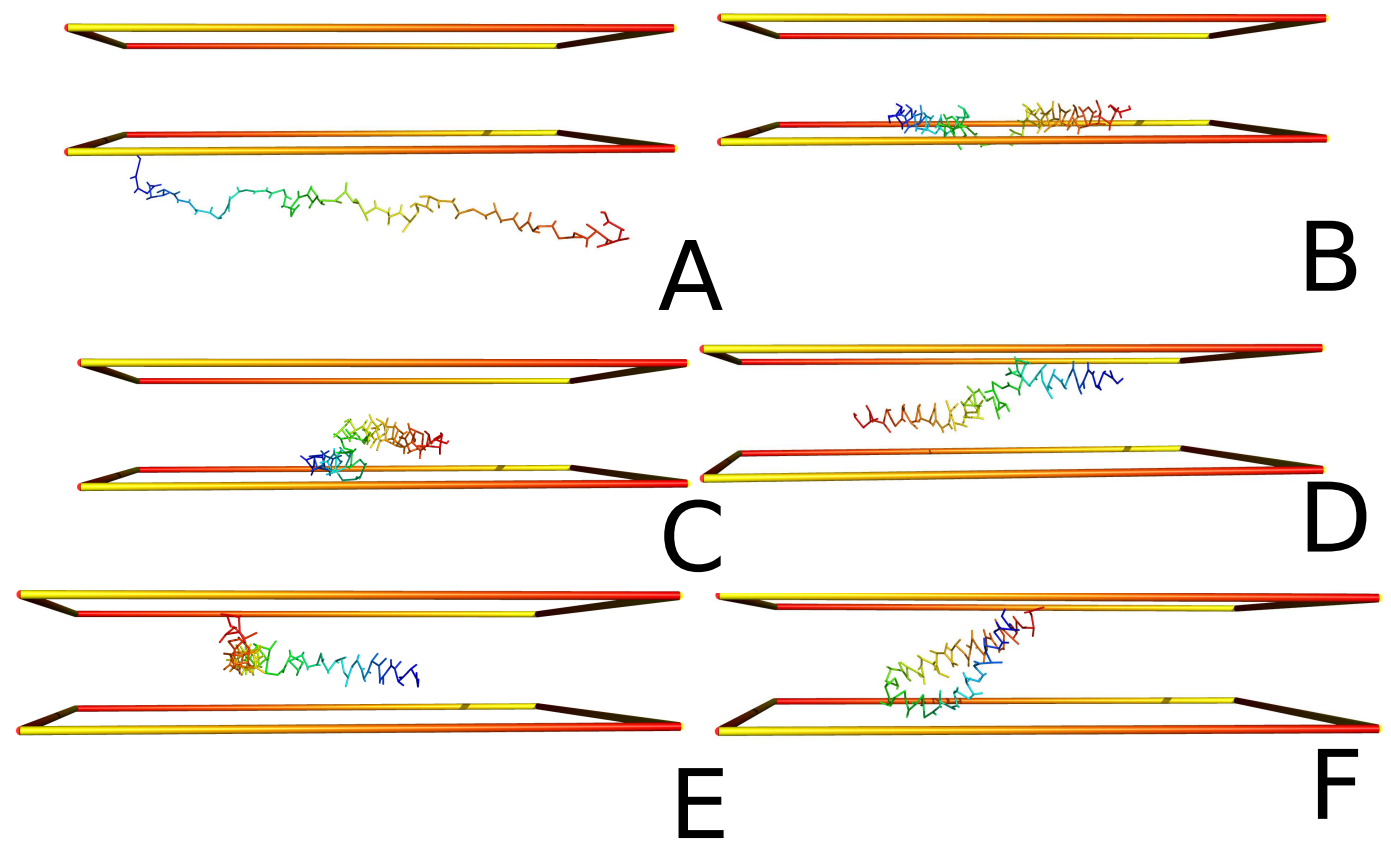

Figure S11. Snapshots from a trajectory of the 1A91 protein simulated folding. 

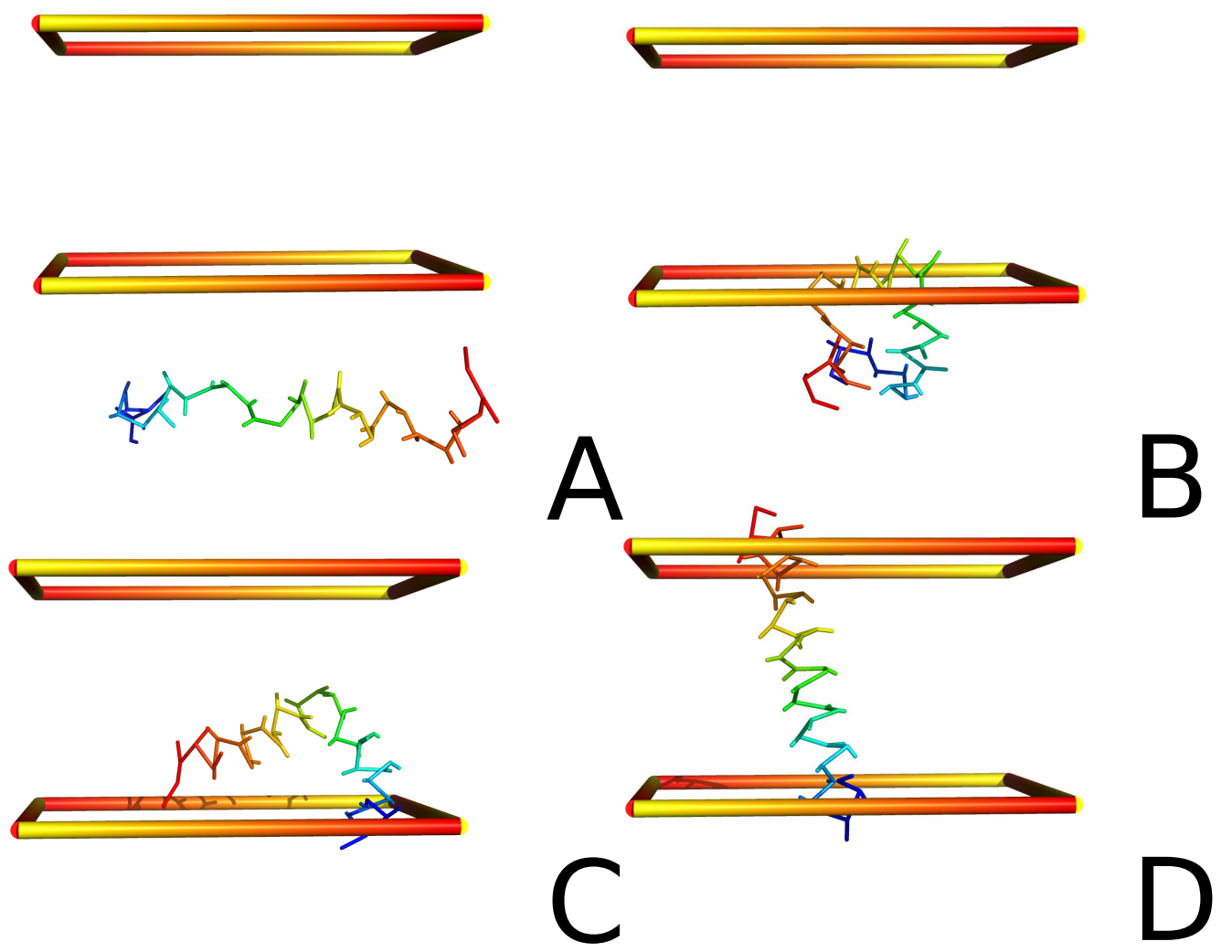

Figure S12. Snapshots from a trajectory of the 1IIJ protein simulated folding. 


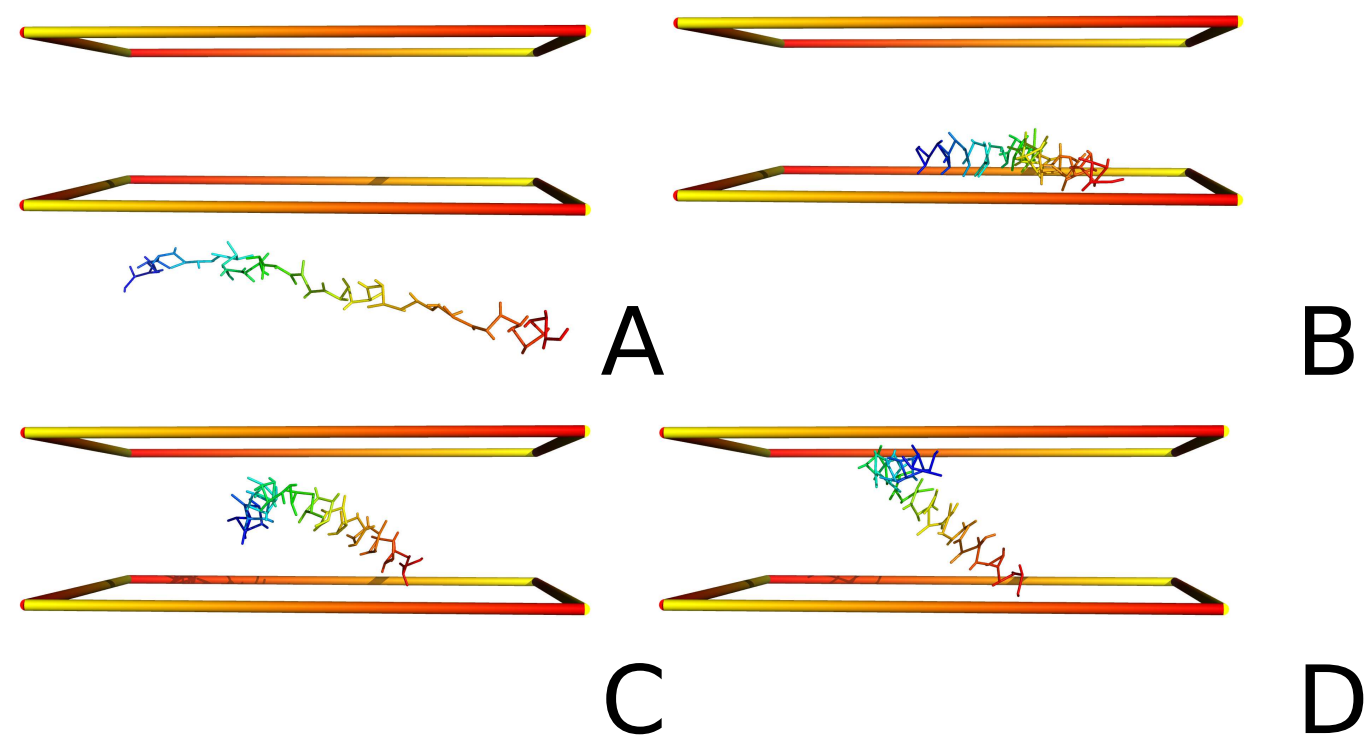

Figure S13. Snapshots from a trajectory of the 1N7L protein simulated folding. 

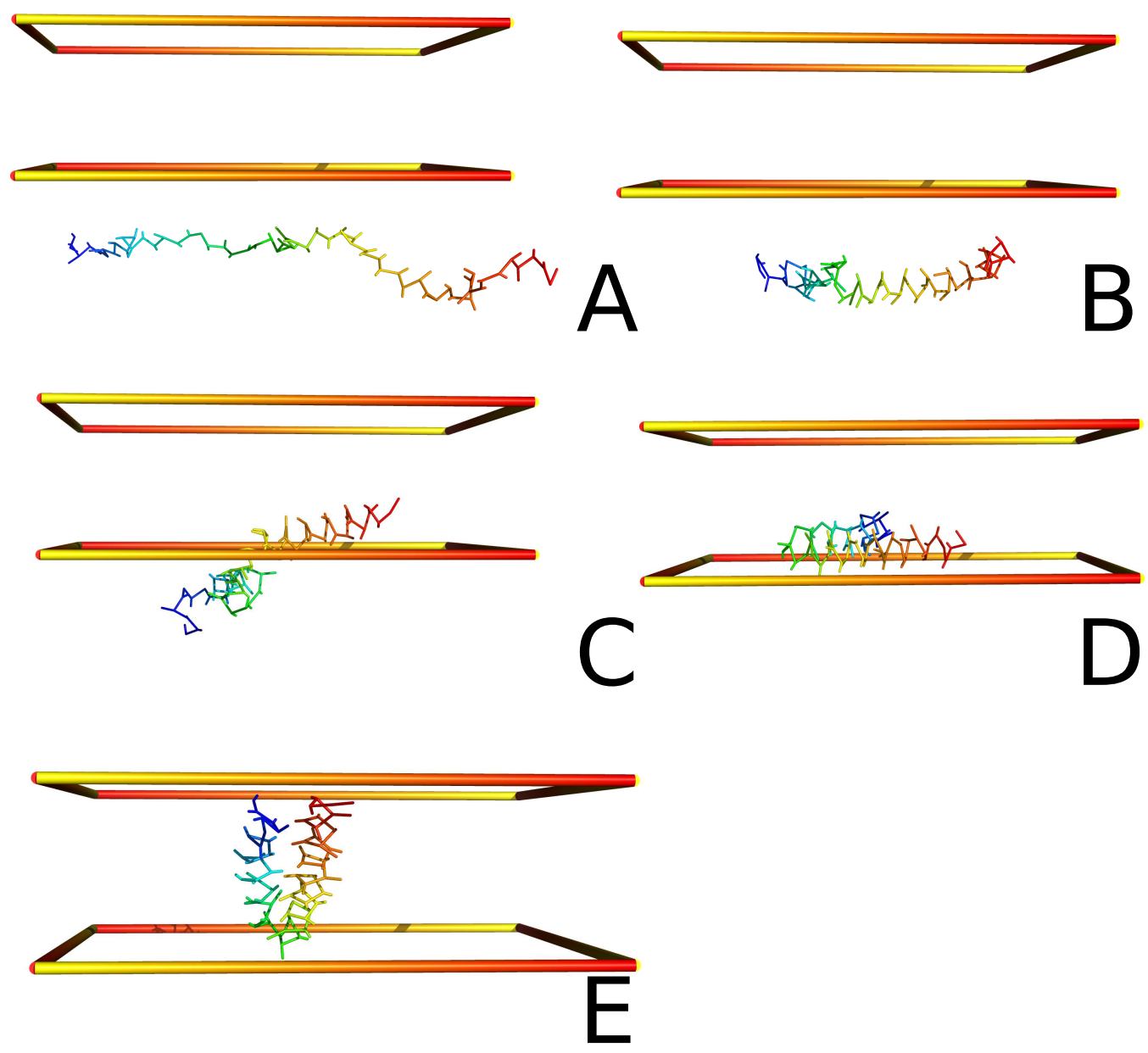

Figure S14. Snapshots from a trajectory of the 1VRY protein simulated folding. 

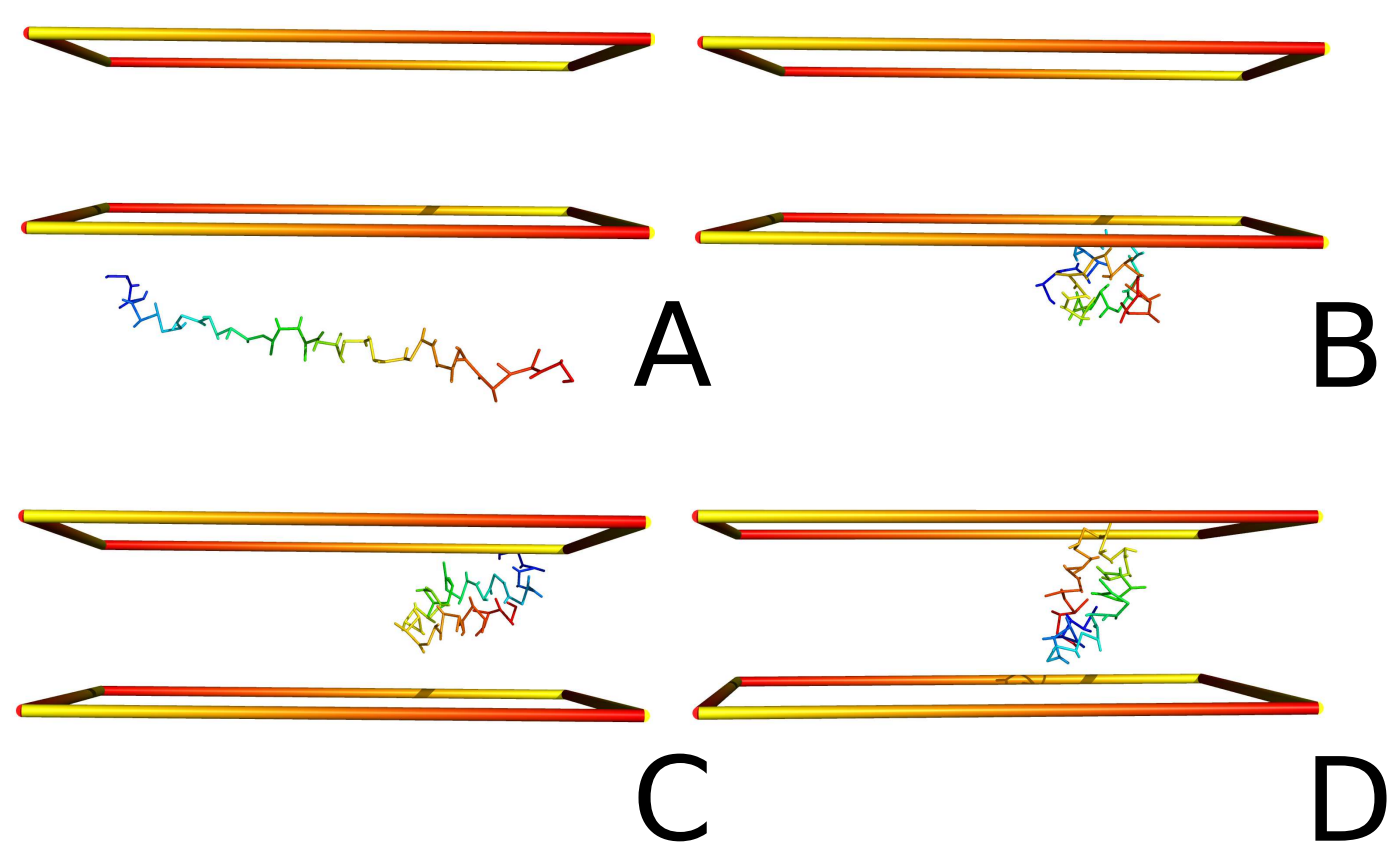

Figure S15. Snapshots from a trajectory of the 1WAZ protein simulated folding. 


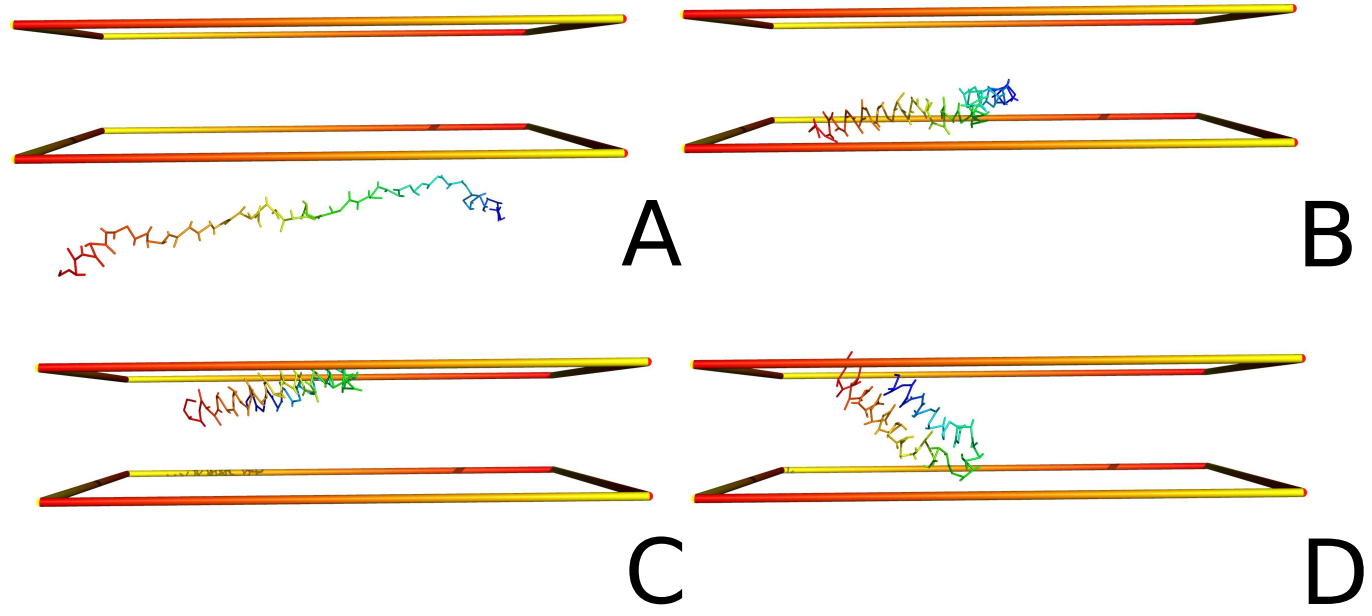

Figure S16. Snapshots from a trajectory of the 1WU0 protein simulated folding. 


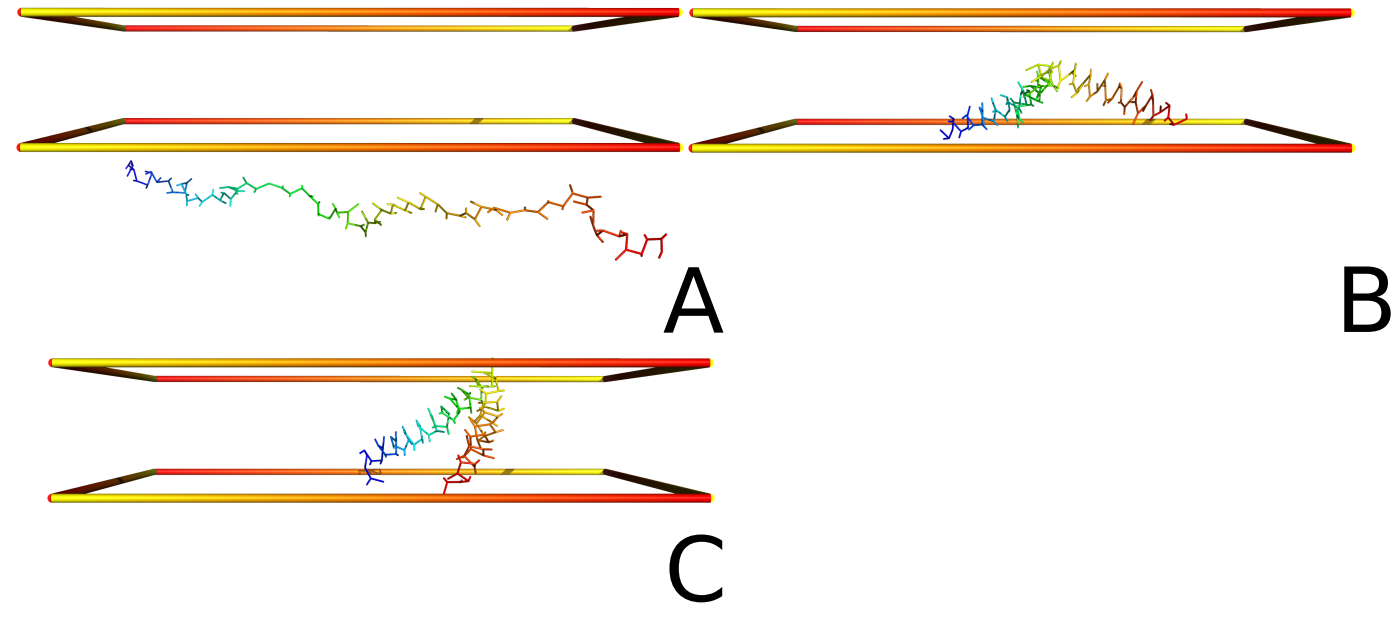

Figure S17. Snapshots from a trajectory of the 2K9P protein simulated folding. 


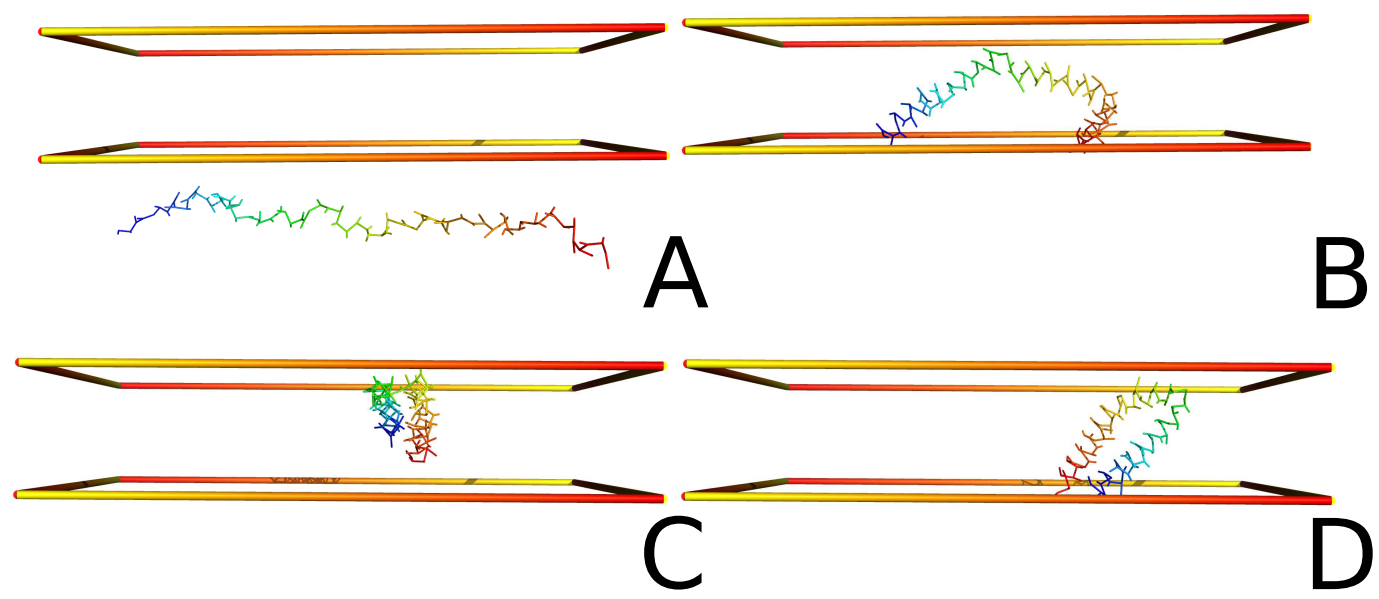

Figure S18. Snapshots from a trajectory of the 2KSD protein simulated folding. 


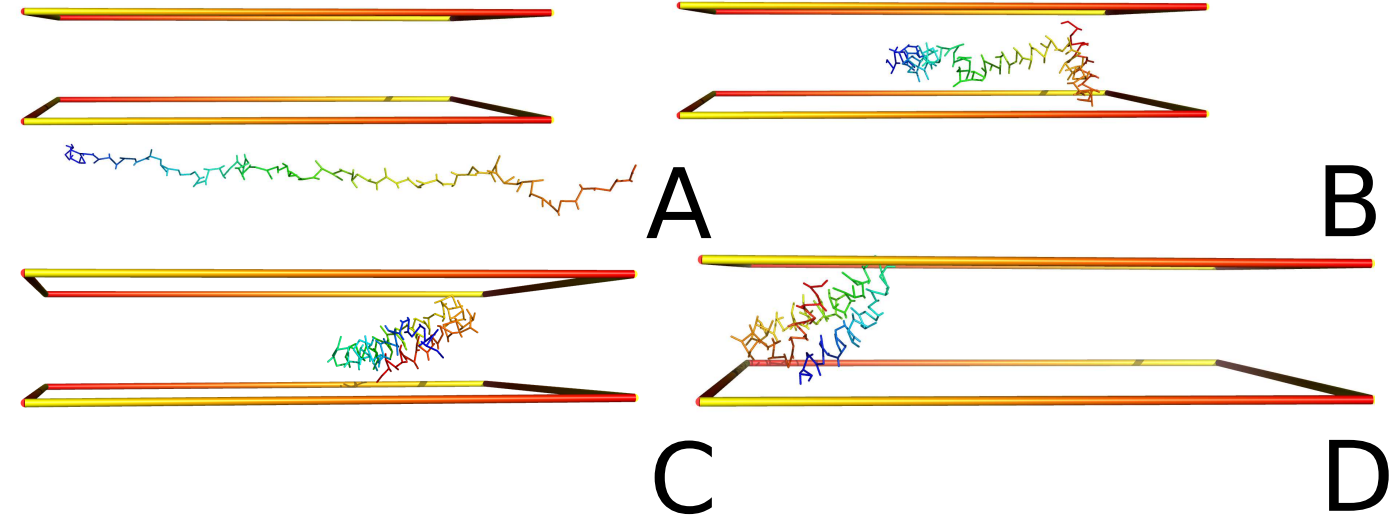

Figure S19. Snapshots from a trajectory of the 2LOP protein simulated folding. 


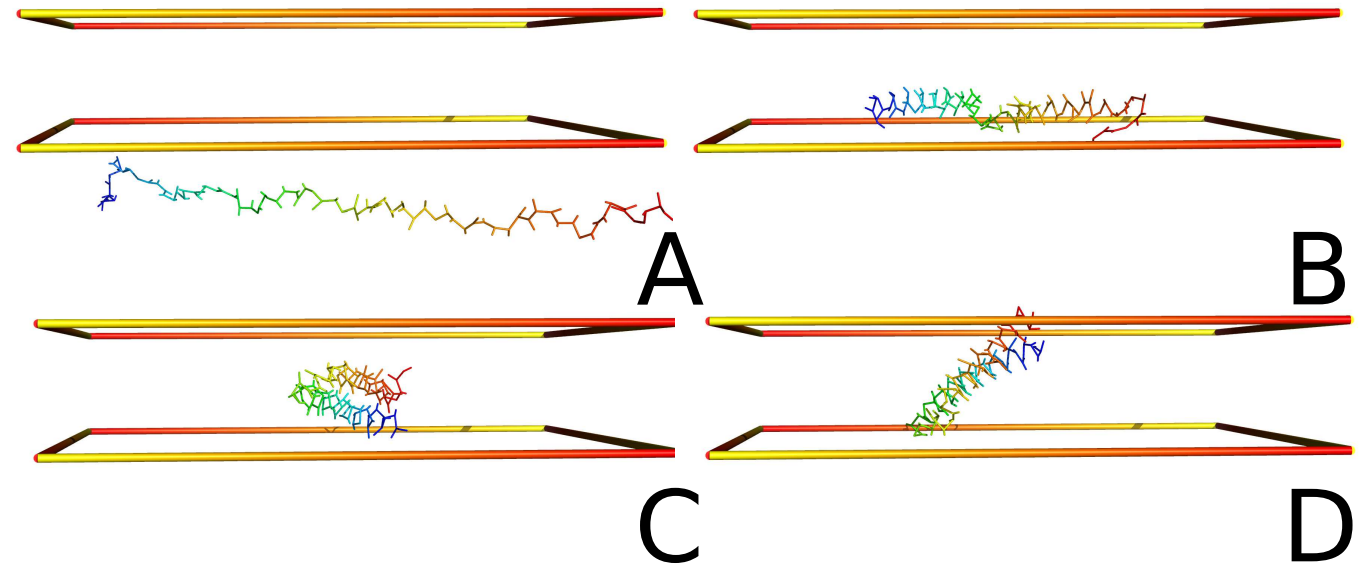

Figure S20. Snapshots from a trajectory of the 2MOZ protein simulated folding. 